\title{
Robust Active Magnetic Bearing Control Using Stabilizing Dynamical Compensators
}

\author{
G.R. Duan, Z.Y. Wu, C.M. Bingham and D. Howe \\ Electrical Machines and Drives Group, Department of Electrical and Electronic Engineering, \\ The University of Sheffield, Mappin Street, Sheffield SI 3JD, UK.
}

\begin{abstract}
The robust control of active magnetic bearings, based on a linearised interval model, is considered. Through robust stability analysis, all the first-order robust stabilizing dynamical compensators for the interval system are obtained. Disturbance attenuation and minimum control effort are also addressed. The approach is applied to a high-speed flywheel supported by two active and two passive magnetic bearings. Simulation and experimental results both show that it is simple, effective, and robust.
\end{abstract}

Keywords: Magnetic bearings, interval systems, dynamical compensators, robust stabilization, disturbance attenuation, minimal control.

\section{INTRODUCTION}

Active magnetic bearings (AMBs) have several advantages over conventional bearings, and are being employed increasingly in a variety of applications [1] However, two difficulties concerning magnetic bearing control involve system uncertainties and the difficulty of obtaining a good velocity signal [2]. In this paper, a basic $\mathrm{AMB}$ comprising two opposing electromagnets and a rigid rotor, as shown in Fig. 1, is considered. To cope with system parameter uncertainties, an interval model is employed. To avoid the use of a velocity feedback signal, a dynamical compensator, which uses only positional information, is utilised for control. By deriving a stability condition for the closed-loop system, a parameterisation of all the robust stabilising dynamical compensators for the interval system is obtained. By appropriately selecting the free parameters in the robust stabilizing controller, the $\mathrm{H}_{2}$ norm of the transfer function from disturbance to output is made arbitrarily small over the system parameter intervals, and the $H_{\infty}$ norm of the controller transfer function is made arbitrarily close to a lower bound. The propesed approach is applied to a high-speed flywheel supported by two active radial magnetic bearings and two passive axial magnetic bearings.

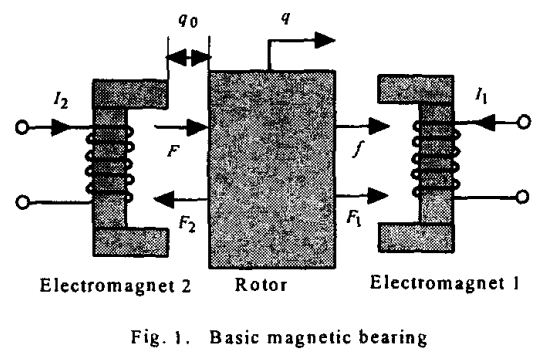

\section{PLANT MODEL AND CONTROLLER}

The dynamical mathematical model for the active magnetic bearing shown in Fig. 1 can be established as:

$m \ddot{q}=\frac{\mu_{0} A N^{2}}{4}\left[\left(\frac{I_{1}}{q_{0}-q}\right)^{2}-\left(\frac{I_{2}}{q_{0}+q}\right)^{2}\right]+f+F$

where $m$ and $q$ are, respectively, the mass $(\mathrm{kg})$ and the position displacement $(\mathrm{m})$ of the rotor; $q_{0}$ is the nominal air gap (m); $\mu_{0}=4 \pi \times 10^{-7} \mathrm{H} / \mathrm{m} ; A$ is the total pole-face area of each electromagnet $\left(\mathrm{m}^{2}\right) ; N$ is the number of turns per coil; $I_{1}, I_{2}$ are the currents in the coils (A); $f$ is an unknown disturbance $(\mathrm{N})$; and $F$ is some known force acting on the rotor $(\mathrm{N})$. When linearised at the equilibrium point $I_{1}=I_{2}=I_{0}, q=0$, with the control diagram shown in Fig. 2, the above model (1) becomes

$$
\ddot{q}-\omega^{2} q=\sigma u+\frac{1}{m}(f+F)
$$

where $\omega$ and $\sigma$ are determined by $I_{0}, q_{0}$, and the system physical parameters, and $u$ is the derived control action. Due to inaccuracies in the measurement of some of the physical parameters and changing environmental conditions, the parameters $\omega$ and $\sigma$ are generally uncertain. Without loss of generality, however, it can be assumed that their values lie within some known intervals, viz.

$$
\omega \in\left[\begin{array}{ll}
\omega_{1} & \omega_{2}
\end{array}\right], \quad \sigma \in\left[\begin{array}{ll}
\sigma_{1} & \sigma_{2}
\end{array}\right]
$$

where $\omega_{1}, \omega_{2}, \sigma_{1}$ and $\sigma_{2}$ are known scalars satisfying

$$
\omega_{2} \geq \omega_{1}>0, \quad \sigma_{1} \leq \sigma_{2}<0
$$

If $y$ is the measured value of $q$, then a general first-order output dynamical compensator for system (2) can be written in the form [3]:

$$
\left\{\begin{array}{l}
\dot{z}=k_{22} z+k_{21} y \\
u=k_{11} y+k_{12} z+k_{f} F
\end{array}\right.
$$


where $k_{i j}, i, j=1,2$, are four scalar controller coefficients, to be designed, and the coefficient $k_{f}$ is given by

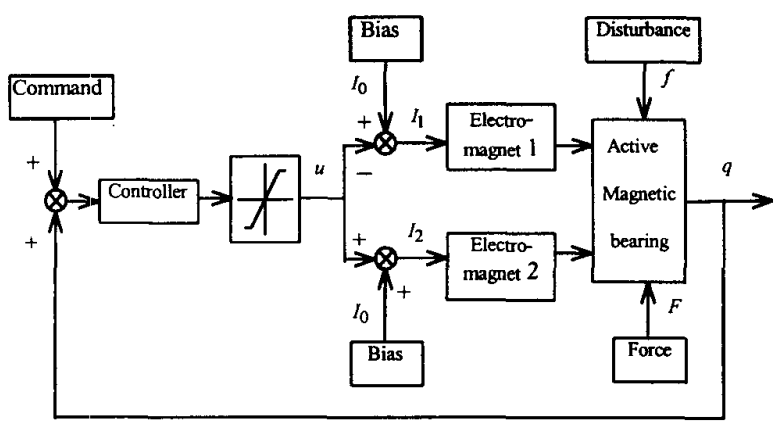

Fig. 2. Control System

$$
k_{f}=-\frac{1}{m \sigma}=\frac{q_{0}^{2}}{\mu_{0} A N^{2} I_{0}}
$$

The resulting closed-loop system can subsequently be written in the state-space form:

$$
\left\{\begin{array}{l}
\dot{\xi}=A_{m c} \xi+d_{m c} f \\
y=c_{m} \xi
\end{array}\right.
$$

with

$$
\begin{gathered}
A_{m c}=\left[\begin{array}{cc}
A_{m}+b_{m} k_{11} c_{m} & b_{m} k_{12} \\
k_{21} c_{m} & k_{22}
\end{array}\right]=\left[\begin{array}{ccc}
0 & 1 & 0 \\
\sigma k_{11}+\omega^{2} & 0 & \sigma k_{12} \\
k_{21} & 0 & k_{22}
\end{array}\right](4 \mathrm{~b}) \\
c_{m c}=\left[\begin{array}{lll}
1 & 0 & 0
\end{array}\right], \quad d_{m c}^{T}=\left[\begin{array}{lll}
0 & \frac{1}{m} & 0
\end{array}\right]
\end{gathered}
$$

\section{ROBUST STABILIZATION}

The dynamical compensator (3) is said to be a robust stabilizing compensator for the interval system (2), or is said to robustly stabilize the interval system (2), if the matrix. $A_{m c}$ given in (4b) is Hurwitz stable for all $\omega$ and $\sigma$ satisfying (2b) and (2c). For robust stabilization of system (2) with the dynamical compensator (3), the following result holds.

Theorem 1: All the robust stabilizing compensators in the form of (3), for system (2), are given by

$$
\left\{\begin{array}{l}
k_{11}=-\frac{1}{\sigma_{2}}\left(\omega_{2}^{2}+\alpha\right) \\
k_{22}=-\beta \\
k_{12}=\gamma \\
k_{21}=\frac{\alpha \beta \tau}{\sigma_{2} \gamma}
\end{array}\right.
$$

with $\alpha, \beta, \gamma$ and $\tau$ being real scalars satisfying

$$
\alpha>0, \beta>0, \gamma \neq 0,0<\tau<1
$$

\section{DISTURBANCE ATTENUATION}

The disturbance attenuation specification can be expressed as follows:

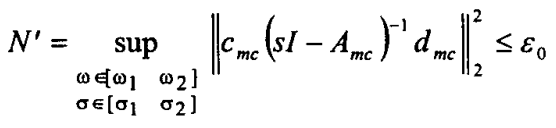

where $\varepsilon_{0}$ is an arbitrary positive number.

Theorem 2: Let $\varepsilon_{0}$ be an arbitrary positive scalar, then, the robust stabilizing dynamical compensator for the interval system (2), given by (3) and (5),

(i) guarantees the specification in (6) if the parameter $\alpha$ is taken as

$$
\alpha=\frac{1+\sqrt{1+8 \varepsilon_{0} \beta^{3} \frac{\tau}{1-\tau}}}{4 \varepsilon_{0} \beta \tau}+\theta^{2}
$$

(ii) guarantees the specification in (6), and minimises the index $N^{\prime}$ with respect to $\beta$, if the parameters $\alpha$ and $\beta$ are taken as

$$
\left\{\begin{array}{l}
\alpha=\frac{1}{\sqrt[3]{\varepsilon_{0}^{2} \tau^{2}(1-\tau)}}+\theta^{2} \\
\beta=\sqrt{\left(\frac{1-\tau}{\varepsilon_{0} \tau}\right)^{\frac{2}{3}}+\theta^{2}(1-\tau)}
\end{array}\right.
$$

(iii) guarantees the specification in (6), and minimises the index $N^{\prime}$ with respect to both $\beta$ and $\tau$, if the parameters $\alpha, \beta$ and $\tau$ are taken as

$$
\left\{\begin{array}{l}
\alpha=3\left(\frac{1}{\sqrt[3]{2 \varepsilon_{0}}}+\theta^{2}\right)^{2} \\
\beta=\frac{1}{\sqrt[3]{2 \varepsilon_{0}}}+\theta^{2} \\
\tau=\frac{2}{3}
\end{array}\right.
$$

where in (7)-(9), $\theta$ is an arbitrary real scalar.

\section{v. MINIMUM CONTROL EFFORT}

The controller given in (3) can be written, in the frequency domain, as follows:

$$
u(s)=G_{u y}(s) y(s), \quad G_{u y}(s)=\left(\frac{k_{12} k_{21}}{s-k_{22}}+k_{11}\right)
$$

In order to facilitate a small control effort, the following (5a) index is proposed:

$$
J=\left\|\frac{k_{12} k_{21}}{s-k_{22}}+k_{11}\right\|_{\infty}
$$


which can be shown to have the greatest lower bound

$$
J_{\mathrm{glb}}=\frac{\omega_{2}^{2}}{\left|\sigma_{2}\right|}
$$

The control effort specification can, therefore, be expressed as

$$
J-\frac{\omega_{2}^{2}}{\left|\sigma_{2}\right|}=\left\|\frac{k_{12} k_{21}}{s-k_{22}}+k_{11}\right\|_{\infty}-\frac{\omega_{2}^{2}}{\left|\sigma_{2}\right|} \leq \varepsilon_{u}
$$

where $\varepsilon_{u}$ is an arbitrarily given positive scalar.

Theorem 3: Let $\varepsilon_{0}$ and $\varepsilon_{u}$ be two arbitrary positive scalars. Then, the robust dynamical stabilizing compensator given by (3) and (5) for the interval system (2), guarantees the specification in (6), and at the same time,

(i) meets the control effort restriction (13) if $\alpha$ is taken as in (7) and $\tau$ is chosen as

$$
\tau=\frac{\beta^{2}+\left|\sigma_{2}\right| \varepsilon_{u}}{\beta^{2}+\left|\sigma_{2}\right| \varepsilon_{u}\left(1+2\left|\sigma_{2}\right| \beta \varepsilon_{0} \varepsilon_{u}\right)}
$$

(ii) meets the control effort restriction (13), and minimises the index $N^{\prime}$ with respect to $\beta$ if

$$
\left\{\begin{array}{l}
\alpha=\frac{1+\varepsilon_{0} \sqrt{\left(\varepsilon_{u}\left|\sigma_{2}\right|\right)^{3}}}{\varepsilon_{0} \sqrt{\varepsilon_{u}\left|\sigma_{2}\right|}}, \quad \beta=\sqrt{\varepsilon_{u}\left|\sigma_{2}\right|} \\
\tau=\frac{1}{1+\varepsilon_{0} \sqrt{\left(\varepsilon_{u}\left|\sigma_{2}\right|\right)^{3}}}
\end{array}\right.
$$

\section{SIMULATION AND EXPERIMENTS}

The proposed approach has been applied to a flywheel energy storage system which is being developed for use in urban electric vehicles. A schematic of the flywheel is shown in Fig.3. The flywheel rim is supported by two active radial magnetic bearings, whose normal air gap is $q_{0}=0.4 \mathrm{~mm}$, and two passive axial bearings. The active bearings have a homopolar topology, each electromagnet comprising two abutting E-shaped cores. On the vertical axis, the pole-face area of each core is $10.4 \times 10^{-4} \mathrm{~m}^{2}$, whilst on the horizontal axis the pole-face area is $5.2 \times 10^{-4} \mathrm{~m}^{2}$. Each core carries a coil having $\mathbf{4 0}$ turns. The mass of the flywheel rim is $12 \mathrm{~kg}$. The upper bounds for the two parameters $\omega$ and $\sigma$ in the linear model (2) for the two electromagnets on the vertical axis were chosen to be $\omega_{2}=390$ and $\sigma_{2}=-4.5$, while those for the electromagnets on the horizontal axis were chosen to be $\omega_{2}=280$ and $\sigma_{2}=-2$. Based on these values and Theorem 3, robust controllers in the form of (3) for the electromagnets on both the vertical and horizontal axes were obtained. The resulting coefficients are shown in Table 1.

Applying controller (3), with the coefficients given in Table 1, to the non-linear system (1) yields the non-linear closed-loop control system. Assuming $m=6 \mathrm{~kg}$ for both the vertical and horizontal axes, simulations of the non-linear closed-loop systems for both axes were carried out, and satisfactory results obtained. The controllers were then implemented digitally on a TMS320C40 DSP-based

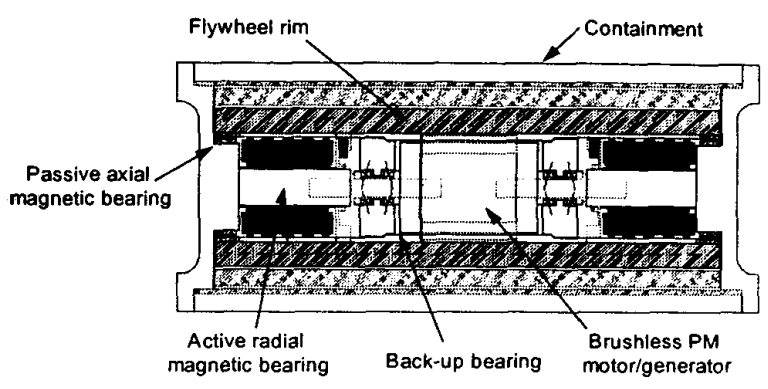

Fig. 3 Schematic of flywheel unit

hardware platform. Various experiments have been carried out to validate their performance. By way of example, Fig.4 shows the initial responses when all four electromagnets on each magnetic bearing are energised simultaneously, $\mathrm{x} 1$ and $\mathrm{x} 2$ being the horizontal position of the flywheel rim, and $y 1$ and $y 2$ being the vertical position. The results clearly demonstrate the stability attributes of the controller for this system. Interestingly, y1 also indicates the presence of a non-minimum phase zero. This is a multi-variable phenomena, and is due to imperfect matching of the speed of response of the bearings at each end of the flywheel rim.

TABLE 1. CONTROLLER COEFFICIENTS

\begin{tabular}{|c|c|c|}
\hline Coefficients & Vertical & Horizontal \\
\hline$k_{11}$ & 256022 & 539200 \\
$k_{12}$ & 10000 & 10000 \\
$k_{21}$ & -111111 & -250000 \\
$k_{22}$ & -5000 & -5000 \\
$k_{f}$ & $1.610880 \mathrm{E}-2$ & $1.610880 \mathrm{E}-2$ \\
\hline
\end{tabular}
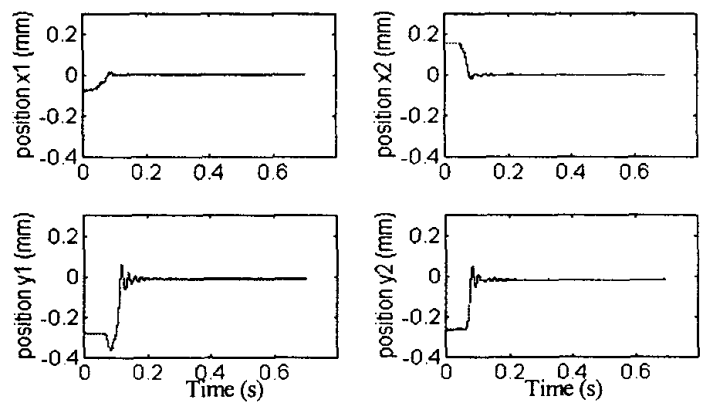

Fig. 4 Initial energisation of flywheel bearing system

\section{REFERENCES}

[1] M. Dussaux, "The industrial applications of active magnetic bearing technology," Proceedings of the Second International Symposium on Magnetic Bearings, July 12-14, 1990, pp.33-38.

[2] J. Levine, J. Lotton, and J.C. Ponsart, "A nonlinear approach to the control of magnetic bearings," IEEE Transactions on Control Systems Technology, vol. 4, no. 5, 1996, pp.524-544

[3] G. R. Duan, "Robust eigenstructure assignment via dynamical compensators," Automatica, vol. 29, no. 2, 1993, pp.469474. 